Sinergias educativas

ISSN: 2661-6661

compasacademico@icloud.com

Grupo Compás

Ecuador

\title{
Los objetos de estudio de la poli\#tica educativa: una tarea para impensar
}

\author{
Acun\#a Gamboa, Luis Alan \\ Los objetos de estudio de la poli\#tica educativa: una tarea para impensar \\ Sinergias educativas, vol. 2, núm. 2, 2017 \\ Grupo Compás, Ecuador \\ Disponible en: http://www.redalyc.org/articulo.oa?id=573561679008
}

Esta obra está bajo una Licencia Creative Commons Atribución-NoComercial-SinDerivar 4.0 Internacional. 


\section{Los objetos de estudio de la poli\#tica educativa: una tarea para impensar}

The objects of study of educational policy: a task to think

Luis Alan Acuña Gamboa acugam2319@gmail.com

Doctor en Estudios Regionales, México

http://orcid.org/0000-0002-8609-4786

Sinergias educativas, vol. 2, núm. 2, 2017

Grupo Compás, Ecuador

Recepción: 15 Marzo 2017

Aprobación: 25 Noviembre 2017

Redalyc: http://www.redalyc.org/ articulo.oa?id $=573561679008$

\section{Obra resen\#ada:}

Tello, Ce\#sar (Compilador). (2015). Los objetos de estudio de la poli\#tica educativa. Hacia una caracterizacio\#n del campo teo\#rico. Buenos Aires: Autores de Argentina.

En los u\#ltimos an\#os, la investigacio\#n educativa se ha concebido como el espacio propicio para el ana\#lisis, la reflexio\#n y el debate, con el fin de medrar la realidad social de los pai\#ses de Ame\#rica Latina y el Caribe. Ante los embates de la era global y consumista que sojuzgan esta regio\#n del mundo, es preciso atreverse a impensar la poli\#tica y las poli\#ticas pu\#blicas educativas que direccionan las acciones de los Sistemas Educativos Latinoamericanos. De esta manera, el libro Los objetos de estudio de la poli\#tica educativa. Hacia la caracterizacio\#n del campo teo\#rico se constituye, precisamente, por la legi\#tima necesidad de abrir nuevos espacios de dia\#logo sobre el campo teo\#rico, epistemolo\#gico y <epistemetodolo\#gico de la Poli\#tica Educativa.

La resen\#a que aqui\# se presenta busca despertar la curiosidad de los lectores, esa curiosidad cienti\#fica que lleve a buscar a\#vidamente esta obra compilada por Ce\#sar Tello, Director de la Red de Estudios Teo\#ricos y Epistemolo\#gicos en Poli\#tica Educativa, que sin duda es y sera\# un referente imprescindible en este campo de estudio. A pesar que el texto, en toda su extensio\#n, es objeto de una lectura y ana\#lisis riguroso y enriquecedor por y para quienes nos interesamos en el tema; este escrito sustenta sus reflexiones en el primer apartado del libro ("Consideraciones teo\#ricas acerca del objeto de estudio de la poli\#tica educativa”), asi\# como en el cierre, esto por considerar, a ti\#tulo personal, que en estos segmentos se encuentra el quid sobre el que convergen todas las construcciones teo\# ricas de los 13 autores que en el texto participan.

\section{LO TEO\#RICO DE LA POLI\#TICA EDUCATIVA}

En un primer momento, Jefferson Mainardes reflexiona el objeto de estudio de la poli\#tica educativa a trave\#s del ana\#lisis de investigaciones y publicaciones realizadas en Brasil y otros pai\#ses. Producto de este trabajo de inteleccio\#n, el autor define como objeto de estudio en el campo, el ana\#lisis de la poli\#tica educativa formulada por el Estado en 
sus diferentes niveles de accio\#n: federal, estatal y municipal; a lo que agregari\#a, personalmente, el a\#mbito internacional.

Mainardes clasifica el ana\#lisis en la investigacio\#n de/sobre poli\#ticas educativas en tres dimensiones que suceden de lo concreto a lo abstracto. En primer lugar, la dimensio\#n investigaciones sobre poli\#ticas y programas se relaciona con todas aquellas acciones investigativas que tienen por objeto la "...formulacio\#n, implementacio\#n, evaluacio\#n de poli\#ticas, resultados y consecuencias" (Tello, 2015, p. 29). En un segundo nivel de ana\#lisis se encuentran las poli\#ticas educativas, en las cuales el investigador se enfoca en el mecanismo y/o mecanismos que entran en juego en el proceso de toma de decisiones y los resultados inmediatos. Por u\#ltimo, el estudio de las poli\#ticas de la educacio\#n se erige como el cli\#max de las investigaciones teo\#rico-epistemolo\#gicas en este campo, puesto que se pretende identificar "...las estructuras y determinantes ma\#s generales de las "poli\#ticas de la educacio\#n"” (Tello, 2015, p. 29).

La gran necesidad de fortalecer los estudios de/sobre poli\#ticas educativas se sustenta en una de\#bil conceptualizacio\#n y construccio\#n de conocimiento en el campo de la educacio\#n. Por esta razo\#n, desde las reflexiones de Morais (citado en Tello, 2015), no se puede desarrollar un lenguaje y una estructuracio\#n segura para con la teori\#a educativa. Ante tal problema\#tica, la Red Latinoamericana de Estudios Epistemolo\#gicos en Poli\#tica Educativa (ReLePe), con base en el modelo de ana\#lisis de Ce\#sar Tello, busca preponderar la importancia de la perspectiva epistemolo\#gica y del enfoque epistemetodolo\#gico en toda investigacio\#n que tenga por objeto de estudio las poli\#ticas educativas, sucediendo asi\# el plano descriptivo por niveles ma\#s abstractos de inteleccio\#n.

En los apartados "Investigaciones sobre poli\#ticas y programas", asi\# como en "Poli\#ticas educativas y poli\#ticas de la educacio\#n: mecanismos, lo\#gica de intervencio\#n y generacio\#n de teori\#a”, Mainardes expone - retomando trabajos realizados en el campo- las li\#neas de accio\#n o preguntas de reflexio\#n que los interesados en los estudios epistemolo\#gicos en poli\#ticas educativas deben retomar para encaminar sus pesquisas investigativas. Adema\#s, evidencia que las tres dimensiones son inherentes y necesarias de analizar para desarrollar conocimiento de alto nivel en educacio\#n; aspecto que, como se menciono\# anteriormente, adolece este campo de investigacio\#n.

A manera de conclusio\#n, Jefferson Mainardes asevera que “...la discusio\#n sobre el objeto de la poli\#tica educativa se constituye en una cuestio\#n relevante que requiere ser debatida en el campo en te\#rminos teo\#rico-metodolo\#gicos" (Tello, 2015, p. 39).

En "Los objetos de estudio de la poli\#tica educativa: tres argumentaciones epistemolo\#gicas para su ana\#lisis", Ce\#sar Tello presenta algunas de sus reflexiones en torno al Enfoque de las Epistemologi\#as de la Poli\#tica Educativa (EEPE). Al respecto, el autor sustenta este enfoque en tres componentes: la perspectiva epistemolo\#gica, la posicio\#n epistemolo\#gica y el enfoque 
epistemetodolo\#gico; empero, dentro del texto solamente se abordan las dos primeras.

La perspectiva epistemolo\#gica se define como “...la cosmovisio\#n que el investigador [en poli\#tica educativa] asume para llevar adelante su indagacio\#n...” (Tello, 2015, p. 44). Por otro lado, el posicionaminto epistemolo\#gico alude a "...las corrientes teo\#ricas propias del campo, considerando que las teori\#as sustantivas son aquellas que guardan relacio\#n directa con el contenido empi\# rico y teo\# rico de los datos de la investigacio\#n" (Tello, 2015, p. 44). En otras palabras, el posicionamiento epistemolo\#gico es la construccio\#n de la realidad y la postura que el investigador asume ante ella, postura que se sustentara\# desde una perspectiva epistemolo\#gica ad hoc. De esta manera Tello considera que, tanto el posicionamiento como la perspectiva epistemolo\#gica, no surgen de la nada o, como e\#l mismo nombra, de "cabezas vaci\#as".

Para explicar las posturas sobre las que sustenta sus reflexiones, Ce\#sar Tello aclara que existe una gran diferencia entre las poli\#ticas educativas (en plural) y la poli\#tica educativa. El primer te\# rmino hace referencia a la toma de decisiones poli\#ticas; en otras palabras, se alude a lo concreto -en te\#rminos de Mainardes - de los procesos de investigacio\#n en el campo. Por otro lado, la poli\#tica educativa (en singular), se relaciona con un ana\#lisis ma\#s profundo que supere el plano descriptivo para posicionarse en la inteleccio\#n ma\#s cienti\#fica de los feno\#menos poli\#ticos; es decir, lo abstracto de los estudios en el campo. Asi\#, Tello afirma que "...las poli\#ticas educativas son el objeto de estudio de la poli\#tica educativa” (Tello, 2015, p. 48).

Con base en estas reflexiones se puede afirmar que el estudio de la poli\#tica educativa obliga al investigador a posicionar su trabajo en el plano de las investigaciones transdisciplinares; es decir, sustentar y profundizar su ana\#lisis ma\#s alla\# de las fronteras del campo de la poli\#tica educativa, ya que en esta emigracio\#n disciplinar es “...donde nos encontraremos con las epistemologi\# as de la poli\#tica educativa..." (Tello, 2015, p. 48).

Otro punto medular en el texto se relaciona con la caracterizacio\#n del objeto de estudio de la poli\#tica educativa. Relacionado con esto, el autor comenta que este tipo de estudio no se limita a las mediaciones que se encuentran en el campo educativo; en otras palabras, las Leyes, el Estado, la escuela y las distintas formas de gobierno no son, como tal, su objeto de estudio; ma\#s bien, son las decisiones poli\#ticas en educacio\#n el intere\#s primordial en este campo de conocimiento. Con esto se asevera que el ana\#lisis en investigacio\#n de/sobre poli\#tica educativa debe contemplar todos los contextos, todos los actores que, de una forma u otra, esta\#n en constantes luchas, compromisos, interpretaciones y reinterpretaciones que surgen de "...las distintas arenas poli\#ticas..." (Tello, 2015, p. 58). Dicha postura es una de las conclusiones que Tello hace para este campo de estudio.

Por su parte, Lindomar Wessler Boneti define las poli\#ticas pu\#blicas en te\#rminos de intereses sociales comunes y no en los postulados marxistas de dominacio\#n de clases (sin que esto signifique que se dirima 
la asidua lucha de poder entre estas). De esta manera, las poli\#ticas pu\#blicas son el resultado de ideas o presuposiciones que intentan medrar las necesidades de un contexto determinado, sin que se desestimen los factores que son la rai\#z de dichas ideas; es decir, “...la relacio\#n de intereses, las inserciones ideolo\#gicas, las concepciones cienti\#ficas, las correlaciones de fuerzas sociales, etc." (Tello, 2015, p. 65).

En este sentido, se concibe que las poli\#ticas pu\#blicas educativas son resultado de la imposicio\#n de una visio\#n hegemo\#nica en una localidad o regio\#n determinada; en palabras de Wessler Boneti, son producto del ejercicio de poder simbo\#lico como elemento fundamental del etnocentrismo cienti\#fico, que demarca necesidades y problema\#ticas por resolver y el co\#mo lograrlo, lo cual se resume en la imposocio\#n de objetivos y li\#neas de accio\#n homogeneizadas para las diferentes cotidianidades escolares y pra\#cticas profesionales en las que se accionan: ciencia, fuerza, energi\#a, te\#cnica e infalibilidad son elementos clave de la tradicional hechura de poli\#ticas pu\#blicas (Aguilar Villanueva, 1992) en materia educativa, que le otorgan el estatus de "incuestionables".

Sin embargo, esta postura que sustenta el disen\#o e implementacio\#n de poli\#ticas pu\#blicas educativas, es constantemente cuestionada por grupos insurgentes que buscan, desde las singularidades y diferenciaciones sociales, la veta para el quehacer poli\#tico, abriendo la posibilidad de definir y redefinir al Estado y la Nacio\#n, asi\# como sus funciones respecto a su actuar de cara a las necesidades y demandas sociales regionales. ¿Co\#mo lograr esto cuando los Estados-Nacio\#n — de\#biles de por si\# (Bauman, 2013) - esta\#n sujetos a las relaciones poli\#tico-econo\#micas de las grandes potencias mundiales que, en te\#rminos educativos, buscan una educacio\#n globalizada? Ante tal situacio\#n, grupos y movimientos insurgentes o contrahegemo\#nicos (un claro ejemplo es el Grupo de Trabajo CLACSO Reformas y contrarreformas educativas en Nuestra Ame\#rica), han buscado que se erijan las singularidades, diferencias y la individualidad sobre las intenciones homogeneizadoras del discurso y pra\#ctica hegemo\#nica a nivel mundial; discurso que asevera incluir, pero desde la exclusio\#n social.

Por otro lado, Nicola\#s Betancur concuerda con Ce\#sar Tello al definir a las poli\#ticas (acciones) como la consecuencia de la decisio\#n poli\#tica (intereses) de quienes tienen el poder institucional o social dentro de la vida pu\#blica de un lugar; en otras palabras, las ideas y los actores se conciben como elementos inherentes e "...insoslayables de cualquier indagacio\#n sobre la racionalidad de las poli\#ticas pu\#blicas, entre ellas, las educativas" (Tello, 2015, p. 82), aunque los roles en el campo son diferentes pero complementarios.

Empero, al referir estudios o investigaciones en poli\#ticas pu\#blicas educativas, estas no deben centrarse u\#nicamente en la cu\#spide de la pira\# mide educativa (decisores y hacedores de poli\#tica y poli\#ticas), sino tambie\#n en los actores encargados de implementar dichas decisiones y visiones poli\#ticas al plano de la realidad educativa y contextual de la comunidad estudiantil, actores que no participan activamente en los disen\#os poli\#ticos o, si lo hacen, se limita la expresio\#n a voz 
pasiva; un ejemplo claro de tal situacio\#n son los "pactos, acuerdos y sugerencias" que algunos Organismos Internacionales firman con pai\#ses latinoamericanos que mayor demanda y necesidad de empre\#stitos presentan (Acun\#a Gamboa y Pons Bonals, en prensa); a trave\#s de los cuales se legitima y moviliza el conocimiento “...especializado, en forma de diagno\#stico y propuestas para las poli\#ticas [pu\#blicas] educativas nacionales..." (Tello, 2015, p. 92), lo que conlleva a la imposicio\#n del discurso hegemo\#nico internacional por sobre la realidad local vivida desde los diferentes niveles educativos.

Ante esto, Betancur precisa la necesidad de lograr disen\#os de poli\#ticas pu\#blicas perennes y no distintivas de los grupos y partidos poli\#ticos en turno, poli\#ticas que alberguen en sus entibados la participacio\#n plural de todos los involucrados en el a\#mbito de la educacio\#n a trave\#s de “...'concertaciones', 'planes nacionales', 'consultas' u otros" (Tello, 2015, p. 95). Sin embargo, aunque es una idea que se ha retomado en algunos pai\#ses como Me\#xico, queda de manifiesto que estos espacios de dia\#logo, reflexio\#n y consenso no han funcionado ma\#s que como escaparates de la parafernalia denominada "democracia"(analizar los Foros de Consulta para la revisio\#n del Modelo Educativo de Educacio\#n Ba\#sica que en Me\#xico se realizaron a partir del an\#o 2014); es decir, la apertura de estos espacios responde ma\#s a la obligacio\#n gubernamental por demostrar a la sociedad su "genuino intere\#s" por dar voz a los sujetos que se encuentran en la parte ma\#s baja de la pira\#mide de la decisio\#n poli\#tica, que por el ana\#lisis riguroso de las propuestas expresada por ellos; entonces, ¿sera\# que la disposicio\#n de escenarios como estos son la solucio\#n a los problemas educativos regionales?

Aunque es buena y acertada la opcio\#n que Betancur propone, esta tiende a ser manipulada por los distintos o\#rdenes de gobierno; ma\#s bien se necesitan li\#neas de reflexio\#n emergentes donde se propongan nuevas formas de hacer poli\#tica y poli\#ticas, un espacio de contrarreformas educativas que incidan, desde fuera de lo gubernamental, sobre la realidad educativa latinoamericana; acciones que conduzcan con plena autonomi\# a - no como la del INEE en Me\#xico- sus acciones poli\#ticas educativas desde y para los contextos locales.

DEL CIERRE DEL LIBRO A LA APERTURA DE LA REFLEXIO\#N

Por u\#ltimo, Ce\#sar Tello invita a reflexionar las posibilidades de impensar la Poli\#tica Educativa desde las investigaciones realizadas en el campo, el cual es concebido por el investigador argentino como espacio para "...la escucha, la construccio\#n conjunta y el pensamiento colectivo” (Tello, 2015, p. 240), donde se desea que otras voces levanten el grito con nuevas formas de analizar y reflexionar sobre el campo; asi\#, la ReLePe pretende propiciar lugares de dia\#logo ma\#s alla\# de lo expresado por las "vacas sagradas" en este campo de estudio. Enhorabuena por esta empresa.

Este libro es una invitacio\#n abierta para las generaciones jo\#venes interesadas en el campo de la Poli\#tica Educativa; por ello, lo expuesto 
en las 248 pa\#ginas de esta obra debe ser motivo de ana\#lisis y cri\#tica, jama\#s debe concebirse como los "postulados principales" a seguir. Ce\#sar Tello sentencia que el campo teo\#rico y epistemolo\#gico de la Poli\#tica Educativa necesita del atrevimiento cienti\#fico como medio para la deconstruccio\#n epistemolo\#gica sobre el tema.

Con base en esto, hago extensa la recomendacio\#n de Los objetos de estudio de la poli\#tica educativa. Hacia la caracterizacio\#n del campo teo\#rico, ti\#tulo que, sin lugar a dudas, se concibe como el caldo de cultivo para nuevas miradas y formas de impensar este campo teo\#rico epistemolo\#gico. Sirva la presente resen\#a como medio de difusio\#n de esta gran obra y como la invitacio\#n cordial al debate cri\#tico en este campo de estudio por dema\#s importante en la actualidad regional, donde se privilegia ma\#s la mercantilizacio\#n educativa (Acun\#a Gamboa y Me\#rida Mancilla, en prensa) que la formacio\#n holi\#stica de los individuos.

\section{Referencias}

Acun\#a Gamboa, L. A. y Me\#rida Mancilla, A. (en prensa). Formacio\#n de investigadores educativos en Chiapas: realidades y falacias. En H. Galeano Sandoval y E. Herna\#ndez de la Rosa (Ed.). Procesos y actores educativos en las tensiones del siglo XXI. Puebla, México: Universidad de Oriente.

Acun\#a Gamboa, L. A. y Pon Bonals, L. (2016). La calidad educativa en Me\#xico. De las disposiciones internacionales a los remiendos del proyecto nacional. Revista Internacional de Investigacio\#n en Ciencias Sociales, 12 (2), 155-174.

Aguilar Villanueva, L. F. (1992). La hechura de las poli\#ticaspu\#blicas. Me\#xico: Porru\#a.

Bauman, Z. (2013).La globalizacio\#n. Consecuencias humanas. Me\#xico: Fondo de Cultura Econo\#mica. 